\title{
Temporal Lobe Ependymal Tumor
}

National Cancer Institute

\section{Source}

National Cancer Institute. Temporal Lobe Ependymal Tumor. NCI Thesaurus. Code C131574.

An ependymal tumor affecting the temporal lobe of the brain. 